\title{
Atoms Controlled at the Quantum Limit: Manipulation of Atoms with Laser Light
}

\section{Wolfgang Ertmer and Gerhard Birkl University of Hannover, Germany}

\section{Seldom has the introduction of a new tool revolutionized an already extremely fruitful field more than the application of laser light to atomic physics. Beyond tremendous improvements in spectroscopy, it has initiated the development of a totally new and fast growing branch of physics: laser manipulation of atomic particles. Physicists have moved from being mere observers to become players in the atomic world}

Being able to control the motion of atoms with laser light has initiated a new and growing field in atomic physics.

Unprecedented possibilities for manipulating atoms have been demonstrated. Atoms can be accelerated, decelerated, and stopped. They can be reliably trapped and cooled to temperatures less than a millionth of a Kelvin above absolute zero. At these temperatures manifestations of the wave-like behaviour of massive particles make a new generation of fundamental tests possible and allow the development of a new generation of measurement tools.

Light can exert force on atoms. This fact was known previously to astronomers studying the deflected tails of comets, and could only be put to work in the microscopic world after the development of a bright, highly directional, and spectrally narrow source of light, namely the laser. Shining laser light on atoms can result in accelerations some one-hundred-thousand times stronger than Earth's gravity. But these violent forces can also be tamed in order to cool atoms to temperatures less than a millionth of a Kelvin above absolute zero. Conservative and dissipative forces can be applied almost at will.

The importance of this new branch of physics was recognized by the Royal Swedish Academy of Sciences when they awarded the 1997 Nobel Prize in Physics to Steven Chu (Stanford University), Claude
Below A fluorescing cloud of sodium atoms (centre) is trapped and cooled to a temperature below 1 millikelvin with the help of laser light. (Atom samples with temperatures well below 1 microkelvin can be created.) Wave-like properties of atomic matter can be investigated in detail under these conditions

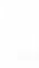

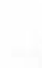

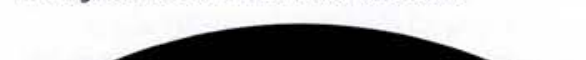
the atom which can result in more than 10 absorption and emission cycles per sec-

ond. In order to guarantee a large rate of scattering events the atomic species has to be selected with respect to having a fast internal transition and a high probability of returning to the initial internal state after the scattering event. Both criteria strongly favour the application of alkali, earth alkali, or metastable noble gas atoms for laser manipulation experiments.

The first experimental investigations consisted of slowing and deflecting atom beams. With a laser beam directed head-on toward an atom beam emerging from a nozzle, the absorption of a photon results in the transfer of one photon momentum against the direction of propagation of the atom. The subsequent re-emission of the photon is isotropic in all directions on average, resulting in no mean momentum transfer. As a net effect the atom is decelerated by one photon momentum per scattering event. In order to fulfil the necessary resonance condition the wavelength of the laser field is chosen in a way to compensate for the Doppler effect due to the fast velocity of the atom. For optimum deceler- 
ation the wavelength of the laser light has to be kept in resonance with the atom's absorption frequency throughout the slowing process. This requires that the change of Doppler shift caused by an atom's changing velocity be compensated for. Two methods have been established for this purpose: Zeeman-slowing relies on the appropriate change in internal resonance frequency caused by a positiondependent magnetic field, whereas in chirp-slowing the laser frequency is changed synchronous with the change in Doppler shift. Applying either of the two methods allows the preparation of an atom beam with very low velocity or even with atoms at rest.

As a second step a slowed beam of atoms can be loaded into a configuration of laser beams with projections on all three components of coordinate space ( $\mathrm{g}$ three pairs of counterpropagating laser beams) and with a frequency of about one natural line width below resonance. In this light configuration moving atoms always see the counterpropagating laser beams shifted closer to resonance and are slowed down by the increased momentum transfer against their direction of motion. Atoms at rest on the other hand see a balanced radiation pressure from all directions allowing only a diffusive motion through the light field. As a result, the atoms move as if being put into a viscous medium, hence the name optical molasses has been adopted for light configuration causing such a strongly damped atom motion. As known from kinetic gas theory an atomic ensemble with a low mean velocity is defined as having a low temperature. Measurements of the temperature achieved in optical molasses gave values typically from $100 \mu \mathrm{K}$ to $1 \mathrm{mK}$ depending on the natural line width of the resonance transition of the atomic species. This temperature is called the Doppler-limit.

\section{Trapping of Atoms}

Even at these low temperatures atoms can diffuse out of the intersection region of the molasses beams. In order to limit atom losses, researchers began to investigate ways to confine atoms to a small region in space for longer periods. The trapping of atoms was put on the agenda and several ideas were found to achieve this goal. Traps for neutral atoms are commonly based on inhomogeneous magnetic fields as well as focused laser beams. In a magnetic field with a local minimum in strength the position dependent Zeeman shift can be used to confine atoms around the potential minimum. In a similar manner the AC-Stark shift experienced by atoms in a focused laser beam of appropriate detuning can also create a local potential minimum. A large detuning from atomic resonance is typically chosen for laser traps in order to suppress spontaneous scattering events causing the unwanted transfer of photon momentum. Both types of traps have been used in a large variety of individual configurations in recent years allowing the storage of atoms with temperatures up to $100 \mathrm{mK}$.

The most commonly used trap for neutral atoms combines stable trapping with the ability to collect and cool atoms in a very efficient way. It is based on an extension of optical molasses. Although in optical molasses only velocity dependent damping forces act; a position-dependent force can be introduced by adding a weak magnetic quadrupole field to the light field. The magnetic field creates a position-dependent shift of the atomic energy levels which is spatially antisymmetric with respect to the zero field point acting as the trap centre. Applying circularly polarized laser light with a slight detuning from atomic resonance results in a position-dependent scattering force. The combination of a three-dimensional magnetic quadrupole field with an optical molasses of six circularly polarized laser beams leads to a position-dependent imbalance in the radiation pressure force on atoms. Atoms outside of the magnetic field zero experience a restoring force towards the trap centre in addition to velocity damping forces. This type of trap is named magneto-optical trap (MOT), a name reflecting the important interplay between magnetic fields and laser light. MOTs are the initial stages of many laser cooling experiments. They allow the trapping of atom samples over many seconds holding up to $10^{10}$ atoms at temperatures well below 1 millikelvin.

\section{The Doppler Limit is not a Limit}

In the late 1980 s experiments with optical molasses allowed the unexpected observation of temperatures far below the Doppler limit which was believed to be the ultimate limit in laser cooling until that time. Then sub-Doppler cooling was discovered. A detailed theoretical investigation showed that in optical molasses the atom population is periodically redistributed between the multiple internal substates of the atomic ground and excited states. Extending the previously accepted model of an atom consisting of only one ground and one excited state to multiple internal states was the key ingredient in understanding this cooling process. As the periodic modulation of the polarization state of the cooling light is crucial, this type of laser cooling was named polarization gradient cooling. With this cooling method caesium atoms for example could be cooled to a temperature of 1 millikelvin more than a factor of 100 below the Doppler-limit. The minimum kinetic energy is limited by the fact that the atoms have to scatter a photon at the end of each cooling cycle and thus pick up a photon momentum.

With this new framework of understanding the interaction of atoms and light fields in more detail even more advanced cooling schemes could be developed: velocity selective coherent population trapping (VSCPT) and Raman cooling both rely on the fact that the atoms can be made to interact with the light field only long enough until they reach a momentum well below the photon momentum. In this instance the interaction with the light field is turned off together with the heating effect due to continuing scattering processes. Applying VSCPT recently allowed temperatures of 180 nanokelvin to be reached for metastable helium atoms, which corresponds to an atom momentum of about one-fifth of a photon momentum.

\section{Wave-like Nature Made Visible}

Although the continuing refinement of laser cooling has led to extremely impressive results in their own right it has also been one of the driving forces in establishing the fields of atom optics and atom interferometry which demonstrate and exploit the wave-like behaviour of atoms. Inherent in the founding principles of quantum mechanics is that massive objects can show particle-like and wavelike behaviour depending on the details of the experiment performed. Since the wavelength of the de Broglie waves corresponding to wave-like atoms are determined by the atom momentum, laser cooled atoms are especially suitable for this type of investigation. In addition, laser manipulation of the external degrees of freedom of atoms is one of the essential tools for studying atom optical effects. In recent years a broad range of atom optical elements have been developed. Evanescent waves have been used to reflect, diffract, and guide atomic matter waves, standing light fields have been applied as beam splitters, focused laser beams as lenses and guiding structures for continued next page 


\section{Metrology with Simple Atoms: Fundamental Constants of Physics}

\section{Massimo Inguscio and Marco Prevedelli University of Florence, Italy}

European laboratories are leading in research where methods of laser spectroscopy are applied to the study of simple atoms. Ultra precise measurements and a comparison with accurate theoretical calculations allow the determination of fundamental quantities such as the Rydberg constant Ry, the fine structure constant $a$ and the r.m.s. charge radius of the proton rp. Stringent tests of QED for bound systems have also been performed

Over the last few years rapid advances in non-linear optics, optical frequency metrology and laser stabilisation techniques have stimulated ultra-high resolution spectroscopic measurements at wavelengths not accessible in the past. Direct

Fig 1 Recording of the two photon transitions on rubidium on which the new optical frequency standard developed in Paris is based

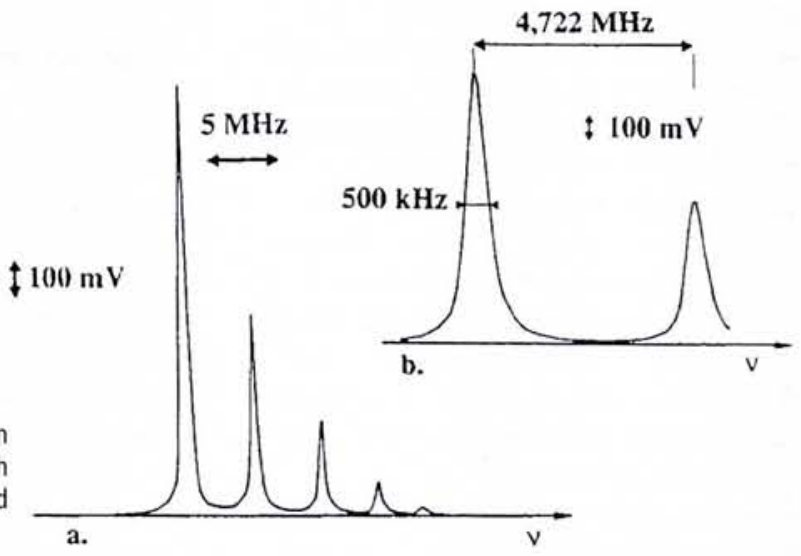

comparison of optical frequencies with the caesium frequency standard can lead to a resolution $\Delta v / v$ of the order of $10^{-13}$. However, these experiments pose formidable technical challenges, and it is only worthwhile to work on them if the frequency for the transitions to be measured can be computed with comparable accuracy from first principles. In that case, most of the amazing experimental accuracy can then be transferred to fundamental constants. Alternatively, the experiment can be considered as a stringent test of theory. The requirement for high theoretical accuracy basically excludes all but the simplest atoms, ie hydrogen and helium.

\section{Hydrogen Experiments}

Recent accurate two-photon spectroscopy on the hydrogen atom reduced the relative uncertainty in the determination of the Rydberg constant $\mathrm{R}_{\mathrm{y}}$, which determines the natural energy scale of atomic and molecular physics, by a factor of 300 over the last two decades. This progress is almost entirely due to efforts by the groups of the Ecole Normal Supérieure in Paris (F. Biraben, B. Cagnac and L. Julien) and at the Max Planck Institute for Quantum Optics in Garching (T.W. Hänsch, previously at Stanford).

In Paris the frequencies of $2 \mathrm{~S}-n \mathrm{~S}$ or $2 \mathrm{~S}$ $n \mathrm{D}$ two-photon transitions with $n$ around 10 , are studied. These transitions conveniently fall within the range of continuous wave Ti:Sapphire lasers. The main advantage of these transitions is that the uncertainty introduced by QED and finite atoms, to name just a few of these developments.

One of the most striking consequences of the wave-like behaviour of atomic matter is the observation of interference effects with atoms. Similar to light in regular optical interferometry an atomic wave packet can take two separate paths from the input port of an atom interferometer to its output port. Depending on the phase difference experienced between the two legs constructive or destructive interference can be observed. A continuous change in the phase difference causes a periodic change between both cases, leading to interference fringes. Besides important studies concerning the very nature of quantum mechanics, atomic physics and quantum optics, atom interferometers are already in use as highly sensitive measurement instruments. In addition to the realization of a new generation of atomic clocks, atom interferometers can be used to study a broad range of external potentials acting on atoms, one example being an interferometer capable of measuring variations in Earth's gravity with a resolution of $\Delta \mathrm{g} / \mathrm{g}$ of the order of $10^{-9}$.

Atomic physics is clearly one of the branches of physics that profited the most from the invention of the laser. The field of controlled manipulation of atoms wouldn't have evolved anywhere close to its present stage if laser light had not been at hand. As described above, laser manipulation is one of the key ingredients in revealing the wave-like behaviour of atomic matter, also manifesting itself so impressively in the observation of Bose-Einstein condensation. A condensate has already been used as a reservoir for a pulsed laser-like source of atoms. The same leap forward that was caused by switching from ordinary light sources to laser light is expected to occur with the development of a continuouswave laser-like source of atoms. Laser manipulation of atoms will have the opportunity of paying back all the benefits it received from regular lasers by developing laser-like atoms sources which may find similarly fruitful applications in other branches of physics.
Further Reading

W.D. Phillips Experiments in Laser Cooling "Fundamental Systems in Quantum Optics" Les Houches Summer School 1990, J. Dalibard, J.-M. Raimond, and J. Zinn-Justin (eds.); Elsevier 1992

C. Cohen-Tannoudji Theory of Laser Cooling "Fundamental Systems in Quantum Optics" Les Houches Summer School 1990, J. Dalibard, J.-M. Raimond, and J. Zinn-Justin (eds.), Elsevier 1992

K. Sengstock and W. Ertmer "Advances in Atomic, Molecular and Optical Physics" vol 35, Academic Press 1995

C.S. Adams, M. Sigel, J. Mlynek Atom Optics and Atom Interferometry Physics Reports vol 240, 1994 